\title{
DARBOUX-LAMÉ EQUATION \\ AND ISOMONODROMIC DEFORMATION
}

MAYUMI OHMIYA

Received 10 September 2002

The Darboux-Lamé equation is defined as the double Darboux transformation of the Lamé equation, and is studied from the viewpoint of the isomonodromic deformation theory. It is shown that the second-order ordinary differential equation of Fuchsian type on $\mathbf{P}_{1}$ corresponding to the second Darboux-Lamé equation is obtained as isomonodromic deformation of some specific Gauss' hypergeometric differential equation.

\section{Introduction}

We consider the $n$th Lamé equation

$$
\frac{\partial^{2}}{\partial x^{2}} f(x)-(n(n+1) \wp(x, \tau)-\lambda) f(x)=0,
$$

where $n$ is a natural number and $\wp(x, \tau)$ is the Weierstrass elliptic function with the fundamental periods 1 and $\tau$ such that $\mathfrak{I} \tau>0$. If the fundamental period $\tau$ and the discrete eigenvalue $\lambda$ satisfy a kind of degenerate condition obtained in [6], we can construct the $n$th algebro-geometric elliptic potential $u_{n, \lambda}^{* *}(x, \xi)$ with the complex parameter $\xi$ by the method of double Darboux transformation. We call the ordinary differential equation

$$
\frac{\partial^{2}}{\partial x^{2}} f(x)-\left(u_{n, \lambda}^{* *}(x, \xi)-\lambda\right) f(x)=0
$$

the $n$th Darboux-Lamé equation of degenerate type. The purpose of the present work is to clarify the isomonodromic property of equation (1.2) regarding $\xi$ as the deformation parameter. Various authors have formerly clarified the isospectral property of the double Darboux transformation (the double commutation) of the $n$th algebro-geometric potential. See, for example, $[2,6]$ and the references therein. However, we could not treat the isomonodromic deformation problems, for $n \geq 3$, in this paper, while the isospectral deformation problem have been almost completely solved for general $n$. 


\section{Preliminaries}

In this section, the necessary materials are summarized. We refer the reader to $[5,6]$ for more precise information.

We consider the second-order linear ordinary differential operator in the complex domain

$$
H(u)=-\frac{\partial^{2}}{\partial x^{2}}+u(x), \quad x \in \mathbb{C},
$$

where $u(x)$ is a meromorphic function. The functions $Z_{n}(u), n \in \mathbb{N}$, defined by the recursion relation

$$
Z_{0}(u) \equiv 1, \quad Z_{n}(u)=\Lambda(u) Z_{n-1}(u), \quad n=1,2, \ldots,
$$

which are the differential polynomials in $u(x)$, are called the KdV polynomials, where

$$
\Lambda(u)=\left(\frac{\partial}{\partial x}\right)^{-1} \cdot\left(\frac{1}{2} u^{\prime}+u \frac{\partial}{\partial x}-\frac{1}{4} \frac{\partial^{3}}{\partial x^{3}}\right)
$$

is the $\Lambda$-operator associated with the differential operator $H(u)$.

Let $V(u)$ be the linear span of all KdV polynomials over $\mathbb{C}$. If $\operatorname{dim} V(u)=n+1$, then $u(x)$ is called the $n$th algebro-geometric potential and we write $\operatorname{rank} V(u)=n$. If $u(x)$ is the $n$th algebro-geometric potential, then there uniquely exist the polynomials $a_{j}(\lambda)$, $j=0,1, \ldots, n$, in the spectral parameter $\lambda$ of degree $n-j+1$ such that

$$
Z_{n+1}(u-\lambda)=\sum_{j=0}^{n} a_{j}(\lambda) Z_{j}(u-\lambda) .
$$

For this fact, see $[5,6]$. The $M$-function $M(x, \lambda ; u)$ associated with $u(x)$ is the differential polynomial defined by

$$
M(x, \lambda ; u)=Z_{n}(u-\lambda)-\sum_{j=1}^{n} a_{j}(\lambda) Z_{j-1}(u-\lambda) .
$$

The spectral discriminant

$$
\Delta(\lambda ; u)=M_{x}(x, \lambda ; u)^{2}-2 M(x, \lambda ; u) M_{x x}(x, \lambda ; u)+4(u(x)-\lambda) M(x, \lambda ; u)^{2}
$$

is the polynomial of degree $2 n+1$ in $\lambda$ with constant coefficients. Let

$$
\operatorname{Spec} H(u)=\{\lambda \mid \Delta(\lambda ; u)=0\},
$$

which corresponds to the discrete spectrum of the operator $H(u)$. If $\lambda_{j} \in \operatorname{Spec} H(u)$, then we have

$$
\left(H(u)-\lambda_{j}\right) M\left(x, \lambda_{j} ; u\right)^{1 / 2}=0
$$

We call $M\left(x, \lambda_{j} ; u\right)^{1 / 2}$ the $M$-eigenfunction. 
For $f(x) \in \operatorname{ker}(H(u)-\lambda) \backslash\{0\}$, the Darboux transformation is the operator $H\left(u^{*}\right)$ with the potential $u^{*}(x)$ defined by

$$
u^{*}(x)=u(x)-2 \frac{\partial^{2}}{\partial x^{2}} \log f(x)
$$

We sometimes call the resulted potential $u^{*}(x)$ itself the Darboux transformation.

Suppose $f(x) \in \operatorname{ker}(H(u)-\lambda) \backslash\{0\}$, then we have

$$
\frac{1}{f(x)} \in \operatorname{ker}\left(H\left(u^{*}\right)-\lambda\right) \backslash\{0\}
$$

This fact is called Darboux's lemma [1]. The Darboux transformation of the algebrogeometric potential $u(x)$ by the corresponding $M$-eigenfunction

$$
u_{\lambda_{j}}^{*}(x)=u(x)-2 \frac{\partial^{2}}{\partial x^{2}} \log M\left(x, \lambda_{j} ; u\right)^{1 / 2}=u(x)-\frac{\partial^{2}}{\partial x^{2}} \log M\left(x, \lambda_{j} ; u\right)
$$

is called the algebro-geometric Darboux transformation (ADT). Let

$$
\widehat{M}\left(x, \lambda_{j} ; u\right)=\int M\left(x, \lambda_{j} ; u\right) d x
$$

and fix the integration constant appropriately; then, by Darboux's lemma, mentioned above, it follows that the function $F_{\lambda_{j}}(x, \xi)$, defined by

$$
F_{\lambda_{j}}(x, \xi)=\frac{\phi_{\lambda_{j}}(\xi)+\xi \widehat{M}\left(x, \lambda_{j} ; u\right)}{M\left(x, \lambda_{j} ; u\right)^{1 / 2}}
$$

is the 1-parameter family of the eigenfunction of $H\left(u_{\lambda_{j}}^{*}\right)$ associated with the eigenvalue $\lambda_{j}$, that is,

$$
\left(H\left(u_{\lambda_{j}}^{*}\right)-\lambda_{j}\right) F_{\lambda_{j}}(x, \xi)=0
$$

where $\phi_{\lambda_{j}}(\xi)$ is an arbitrary function which depends only on $\xi$. The function $\phi_{\lambda_{j}}(\xi)$ will be determined exactly so that the ADDT, which is defined below, of the $n$th Lamé equation is isomonodromic.

The algebro-geometric double Darboux transformation (ADDT) is defined as the Darboux transformation of $u_{\lambda_{j}}^{*}(x)$ by $F_{\lambda_{j}}(x, \xi)$ :

$$
\begin{aligned}
u_{\lambda_{j}}^{* *}(x, \xi) & =u_{\lambda_{j}}^{*}(x)-2 \frac{\partial^{2}}{\partial x^{2}} \log F_{\lambda_{j}}(x, \xi) \\
& =u(x)-2 \frac{\partial^{2}}{\partial x^{2}} \log \left(\phi_{\lambda_{j}}(\xi)+\xi \widehat{M}\left(x, \lambda_{j} ; u\right)\right)
\end{aligned}
$$

In what follows, we assume that $\phi_{\lambda_{j}}(\xi)$ does not identically vanish since $\phi_{\lambda_{j}}(\xi) \equiv 0$, then the $\operatorname{ADDT} u_{\lambda_{j}}^{* *}(x, \xi)$ does not depend on $\xi$. 
Let

$$
\operatorname{Spec}_{m} H(u)=\left\{\lambda_{j} \mid \text { the multiple roots of } \Delta(\lambda ; u)=0\right\}
$$

which we call the multiple spectrum of $H(u)$. It is shown in [6] that if $u(x)$ is the $n$th algebro-geometric potential, then $u_{\lambda_{j}}^{*}(x)$ is the $(n-1)$ th algebro geometric potential if and only if $\operatorname{Spec}_{m} H(u) \neq \varnothing$ and $\lambda_{j} \in \operatorname{Spec}_{m} H(u)$.

If $n$ is a natural number, then the $n$th Lamé potential $u_{n}(x, \tau)=n(n+1) \wp(x, \tau)$ is known to be the $n$th algebro-geometric potential (see, e.g., [5]).

Let $M_{n}(x, \lambda, \tau)$ be the $M$-function associated with the $n$th Lamé potential $u_{n}(x, \tau)$, that is, $M_{n}(x, \lambda, \tau)=M\left(x, \lambda ; u_{n}(x, \tau)\right)$. Let

$$
D\left(\tau ; u_{n}\right)=R\left(\Delta\left(\lambda ; u_{n}\right), \frac{d}{d \lambda} \Delta\left(\lambda ; u_{n}\right)\right)
$$

where $R(P, Q)$ is the resultant of polynomials $P(\lambda)$ and $Q(\lambda)$. If $D\left(\tau_{*} ; u_{n}\right)=0$ for $\tau_{*} \in H^{+}$, then there exists $\lambda_{*} \in \operatorname{Spec}_{m} H\left(u_{n}\right)$, that is, $\lambda_{*}$ is the multiple root of $\Delta\left(\lambda ; u_{n}\right)=0$ and

$$
\operatorname{rank} u_{n, \lambda_{*}}^{*}\left(x, \tau_{*}\right)=n-1
$$

where $u_{n, \lambda_{*}}^{*}(x)$ is the Darboux transformation of the $n$th Lamé potential $u_{n}(x)$ by the corresponding $M$-eigenfunction $M_{n}\left(x, \lambda_{*}, \tau_{*}\right)^{1 / 2}$, that is,

$$
u_{n, \lambda_{*}}^{*}\left(x, \tau_{*}\right)=u_{n}\left(x, \tau_{*}\right)-\frac{\partial^{2}}{\partial x^{2}} \log M_{n}\left(x, \lambda_{*}, \tau_{*}\right) .
$$

Let

$$
\Theta_{n}=\left\{\tau \mid D\left(\tau ; u_{n}\right)=0\right\} \subset H^{+}
$$

and we call it the lattice of degenerate periods associated with the $n$th Lamé potential $u_{n}(x)$. One can immediately see that the lattice of degenerate periods $\Theta_{n}$ is the discrete subset of $\mathrm{H}^{+}$.

Now, we enumerate several examples of the degenerate condition for the Lamé potentials. For this purpose, we must carry out elementary but very complicated computation. Hence, here we explain only the simplest case $n=1$. See also [3] for another method of computation.

KdV polynomials. We have

$$
Z_{0}\left(u_{n}\right)=1, \quad Z_{1}\left(u_{n}\right)=\frac{1}{2} u_{n}, \quad Z_{2}\left(u_{n}\right)=\frac{3}{8} u_{n}^{2}-\frac{1}{8} u_{n}^{\prime \prime} .
$$

Computation of the $M$-function $M_{2}(x, \lambda, \tau)$. Let $\rho_{0}$ and $\rho_{1}$ be the constants such that

$$
\begin{aligned}
Z_{2}\left(u_{1}\right) & =\left(\frac{3}{8}\right)\left(4 \wp^{2}\right)-\left(\frac{1}{8}\right)\left(2 \gamma^{\prime \prime}\right) \\
& =\rho_{0} Z_{0}\left(u_{1}\right)+\rho_{1} Z_{1}\left(u_{1}\right)=\rho_{0}+\rho_{1} \wp
\end{aligned}
$$


Hence, $\rho_{1}=0$ and $\rho_{0}=(1 / 8) g_{2}$ follow immediately. On the other hand, according to [5, Theorem 3, page 414], define the coefficients $\alpha_{\nu}^{(n)}, v=0,1,2, \ldots, n$, for $n=0,1,2$, then we immediately have

$$
\alpha_{1}^{(2)}=\frac{3}{2}, \quad \alpha_{0}^{(2)}=1, \quad \alpha_{0}^{(1)}=\frac{1}{2}, \quad \alpha_{1}^{(1)}=\alpha_{0}^{(0)}=1
$$

Moreover, by [5, Lemma 7, page 417], we have

$$
Z_{2}\left(u_{1}-\lambda\right)=a_{1}(\lambda) Z_{1}\left(u_{1}-\lambda\right)+a_{0}(\lambda) Z_{0}\left(u_{1}-\lambda\right)
$$

where

$$
\begin{gathered}
a_{0}(\lambda)=-\alpha_{0}^{(2)} \lambda^{2}+\alpha_{0}^{(1)} \rho_{1} \lambda+\alpha_{0}^{(0)} \rho_{0}=-\lambda^{2}+\frac{1}{8} g_{2}, \\
a_{1}(\lambda)=-\alpha_{1}^{(2)} \lambda+\alpha_{1}^{(1)}=-\frac{3}{2} \lambda .
\end{gathered}
$$

Hence, we have

$$
M_{1}(x, \lambda, \tau)=\frac{1}{2}(2 \wp-\lambda)-\left(-\frac{3}{2} \lambda\right)=\wp+\lambda
$$

Computation of the spectral discriminant $\Delta\left(\lambda ; u_{1}\right)$. Using the $M$-function $M_{1}(x, \lambda, \tau)$ obtained above, we have

$$
\Delta\left(\lambda ; u_{1}\right)=\wp^{\prime 2}-2(\wp+\lambda) \wp^{\prime \prime}+4(2 \wp-\lambda)(\wp+\lambda)^{2}=-4 \lambda^{3}+g_{2} \lambda-g_{3} .
$$

For the first Lamé potential $u_{1}(x, \tau)$, since $g_{2}(\tau)^{3}-27 g_{3}(\tau)^{2} \neq 0$ for any $\tau \in H^{+}$, $\operatorname{Spec}_{m} H\left(u_{1}\right)=\varnothing$ holds for any $\tau \in H^{+}$, that is, $\Theta_{1}=\varnothing$.

On the other hand, for the second Lamé potential $u_{2}(x, \tau)$, we can compute the spectral discriminant similarly to the above example, and

$$
\Delta\left(\lambda ; u_{2}\right)=-4\left(\lambda^{2}-3 g_{2}(\tau)\right)\left(\lambda^{3}-\frac{9}{4} g_{2}(\tau) \lambda-\frac{27}{4} g_{3}(\tau)\right)
$$

follows. Hence $\operatorname{Spec}_{m} H\left(u_{2}\right) \neq \varnothing$ holds if and only if $g_{2}(\tau)=0$. Note that $g_{2}(\tau)=0$ holds if and only if $J(\tau)=0$, where $J(\tau)=g_{2}(\tau)^{3} /\left(g_{2}(\tau)^{3}-27 g_{3}(\tau)^{2}\right)$ is the elliptic modular function. Since $g_{2}\left(e^{2 \pi i / 3}\right)=0$, by the modular invariance of $J(\tau)$, we have

$$
\begin{aligned}
\Theta_{2} & =\left\{\tau \mid \tau=\frac{a e^{2 \pi i / 3}+b}{c e^{2 \pi i / 3}+d},\left(\begin{array}{ll}
a & b \\
c & d
\end{array}\right) \in \operatorname{SL}(2, \mathbb{Z})\right\} \subset H^{+} \\
& =\{\tau \mid \mathfrak{I} \tau>0\} .
\end{aligned}
$$

\section{The second Darboux-Lamé equation}

Suppose that $\tau_{*} \in \Theta_{n}$ and $\lambda_{*} \in \operatorname{Spec}_{m} H\left(u_{n}\left(x, \tau_{*}\right)\right)$. Let $M_{n}\left(x, \lambda_{*}, \tau_{*}\right)$ be the $M$-function corresponding to the $n$th Lamé potential $u_{n}\left(x, \tau_{*}\right)$. By (2.15), the ADDT of $u_{n}\left(x, \tau_{*}\right)$ is 
expressed as

$$
u_{n, \lambda_{*}}^{* *}(x, \xi)=u_{n}\left(x, \tau_{*}\right)-2 \frac{\partial^{2}}{\partial x^{2}} \log \left(\phi_{\lambda_{*}}(\xi)+\xi \widehat{M}_{n}\left(x, \lambda_{*}, \tau_{*}\right)\right)
$$

where $\widehat{M}_{n}\left(x, \lambda_{*}, \tau_{*}\right)$ is defined by $(2.12)$. We call the 1-parameter family of the ordinary differential equation (1.2) with the potential $u_{n, \lambda_{*}}^{* *}(x, \xi)$, defined above, the $n$th DarbouxLamé equation of degenerate type, and $\mathrm{DL}_{n}\left(\tau_{*}, \lambda_{*}, \xi\right)$ denotes that 1-parameter family (1.2).

In what follows, we construct exactly the second Darboux-Lamé equation of degenerate type. Suppose that $\tau_{*} \in \Theta_{2}$, then, by the direct calculation parallel to that for $M_{1}(x$, $\lambda, \tau)$, we have

$$
M_{2}\left(x, \lambda, \tau_{*}\right)=\lambda^{2}+3 \wp\left(x, \tau_{*}\right) \lambda+9 \wp\left(x, \tau_{*}\right)^{2} .
$$

Since we have shown $g_{2}\left(\tau_{*}\right)=0$ in Section 2,

$$
\Delta\left(\lambda ; u_{2}\right)=-4 \lambda^{2}\left(\lambda^{3}-\frac{27}{4} g_{3}\left(\tau_{*}\right)\right)
$$

follows. Hence $\operatorname{Spec}_{m} H\left(u_{2}\left(x, \tau_{*}\right)\right)=\{0\}$ and we have

$$
M_{2}\left(x, 0, \tau_{*}\right)^{1 / 2}=3 \wp\left(x, \tau_{*}\right) \in \operatorname{ker} H\left(u_{2}\right) .
$$

Therefore, the $\mathrm{ADT} u_{2,0}^{*}(x)$ is given by

$$
u_{2,0}^{*}(x)=2 \wp\left(x, \tau_{*}\right)-\frac{2 g_{3}\left(\tau_{*}\right)}{\wp\left(x, \tau_{*}\right)^{2}}
$$

and, by Darboux's lemma, we have

$$
\frac{1}{M_{2}\left(x, 0, \tau_{*}\right)^{1 / 2}}=\frac{1}{3 \wp\left(x, \tau_{*}\right)} \in \operatorname{ker} H\left(u_{2,0}^{*}\right) .
$$

On the other hand, we have

$$
\widehat{M}_{2}\left(x, 0, \tau_{*}\right)=\int M_{2}\left(x, 0, \tau_{*}\right) d x=\int 9 \wp\left(x, \tau_{*}\right)^{2} d x=\frac{3}{2} \wp^{\prime}\left(x, \tau_{*}\right) .
$$

Hence, by (3.1),

$$
u_{2,0}^{* *}\left(x, \tau_{*}\right)=u_{2}(x)-2 \frac{\partial^{2}}{\partial x^{2}} \log \left(\phi_{0}(\xi)+\frac{3}{2} \xi \wp^{\prime}(x, \tau)\right)
$$

follows. Thus we have the following lemma.

Lemma 3.1. The second Darboux-Lamé equation of degenerate type is explicitly expressed as

$$
\frac{\partial^{2}}{\partial x^{2}} f(x)=\frac{6 \wp\left(x, \tau_{*}\right)\left(\phi_{0}(\xi)^{2}-3 \xi \phi_{0}(\xi) \wp^{\prime}(x, \tau)+(27 / 4) g_{3}\left(\tau_{*}\right) \xi^{2}\right)}{\left(\phi_{0}(\xi)+(3 / 2) \xi \wp^{\prime}\left(x, \tau_{*}\right)\right)^{2}} f(x) .
$$


Moreover,

$$
F_{0}(x, \xi)=\frac{\phi_{0}(\xi)+(3 / 2) \xi \wp^{\prime}\left(x, \tau_{*}\right)}{3 \wp\left(x, \tau_{*}\right)} .
$$

The isospectral property of the potential $u_{2,0}^{* *}\left(x, \tau_{*}\right)$ will be discussed in the forthcoming paper [7].

\section{The Fuchsian equation on $P_{1}$}

Suppose that $\tau_{*} \in \Theta_{2}$, that is, $g_{2}\left(\tau_{*}\right)=0$. Since $g_{2}\left(\tau_{*}\right)^{3}-27 g_{3}\left(\tau_{*}\right)^{2} \neq 0, g_{3}\left(\tau_{*}\right) \neq 0$ follows. In what follows, we fix one of the square roots of $g_{3}\left(\tau_{*}\right)$ and denote it by $\gamma$, that is, $\gamma^{2}=g_{3}\left(\tau_{*}\right)$. Then, by the variable transformation

$$
z=\frac{1}{2 i \gamma} \wp^{\prime}\left(x, \tau_{*}\right)+\frac{1}{2}
$$

the second Darboux-Lamé equation $\mathrm{DL}_{2}\left(\tau_{*}, 0, \xi\right)$ is transformed to the second-order ordinary differential equation of Fuchsian type on $\mathbf{P}_{1}$ :

$$
z(z-1) \frac{\partial^{2}}{\partial z^{2}} \hat{f}(z, \xi)+\left(\frac{4}{3} z-\frac{2}{3}\right) \frac{\partial}{\partial z} \hat{f}(z, \xi)=\Gamma\left(z, \frac{3 i \gamma \xi-2 \phi_{0}(\xi)}{6 i \gamma \xi}\right) \hat{f}(z, \xi)
$$

with the parameter $\xi$, where

$$
\Gamma(x, s)=\frac{2}{3} \frac{(2 s-1) z+s(s-2)}{(z-s)^{2}}, \quad \hat{f}(z, \xi)=f(x, \xi) .
$$

We denote the 1-parameter family of the ordinary differential equation (4.2) by $\widehat{\mathrm{DL}}_{2}\left(\tau_{*}\right.$, $0, \xi)$. The regular singular points of equation (4.2) are

$$
z=0,1, \infty, \quad s=s(\xi)=\frac{3 i \gamma \xi-2 \phi_{0}(\xi)}{6 i \gamma \xi}
$$

In what follows, we assume that $\phi_{0}(0) \neq 0$. Then it follows that $s(0)=\infty$, and that the differential equation $\widehat{\mathrm{DL}}_{2}\left(\tau_{*}, 0,0\right)$ coincides with the hypergeometric equation

$$
z(z-1) \frac{\partial^{2}}{\partial z^{2}} \hat{f}(z)+\left(\frac{4}{3} z-\frac{2}{3}\right) \frac{\partial}{\partial z} \hat{f}(z)=\frac{2}{3} \hat{f}(z)
$$

Now, we construct the fundamental system of solutions of $\widehat{\mathrm{DL}}_{2}\left(\tau_{*}, 0, \xi\right)(4.2)$. Suppose $\xi \neq 0$. Then, by Darboux's lemma and (3.1),

$$
\begin{aligned}
f_{1}(x, \xi) & =\frac{1}{F_{0}(x, \xi)}=\frac{6 \wp\left(x, \tau_{*}\right)}{2 \phi_{0}(\xi)+3 \xi \wp^{\prime}\left(x, \tau_{*}\right)} \\
f_{2}(x, \xi) & =f_{1}(x, \xi) \int \frac{1}{f_{1}(x, \xi)^{2}} d x \\
& =\frac{\wp\left(x, \tau_{*}\right)}{6\left(2 \phi_{0}(\xi)+3 \xi \wp^{\prime}\left(x, \tau_{*}\right)\right)} \int \frac{\left(2 \phi_{0}(\xi)+3 \xi \wp^{\prime}\left(x, \tau_{*}\right)\right)^{2}}{\wp\left(x, \tau_{*}\right)^{2}} d x
\end{aligned}
$$


are the fundamental system of solutions of the second Darboux-Lamé equation

$$
H\left(u_{2,0}^{* *}(x, \xi)\right) f(x, \xi)=-\frac{\partial^{2}}{\partial x^{2}} f(x, \xi)+u_{2,0}^{* *}(x, \xi) f(x, \xi)=0
$$

such that $W\left[f_{1}, f_{2}\right]=f_{1} f_{2 x}-f_{1 x} f_{2}=1$. By the variable transformation (4.1), we have

$$
\wp\left(x, \tau_{*}\right)=\gamma^{2 / 3} z^{1 / 3}(1-z)^{1 / 3}, \quad \wp^{\prime}\left(x, \tau_{*}\right)=i \gamma(2 z-1) .
$$

Let $\hat{f}_{j}(z, \xi)=f_{j}(x, \xi), j=1,2$. We immediately have

$$
\hat{f}_{1}(z, \xi)=\frac{6 \gamma^{2 / 3} z^{1 / 3}(1-z)^{1 / 3}}{2 \phi_{0}(\xi)+3 i \gamma \xi(2 z-1)} .
$$

Similarly, we have

$$
\hat{f}_{2}(z, \xi)=\frac{i z^{1 / 3}(1-z)^{1 / 3}}{18 \gamma\left(2 \phi_{0}(\xi)+3 i \gamma \xi(2 z-1)\right)} \int \frac{\left(2 \phi_{0}(\xi)+3 i \gamma \xi(2 z-1)\right)^{2}}{z^{4 / 3}(1-z)^{4 / 3}} d z .
$$

\section{Isomonodromic property of $\widehat{\mathrm{DL}}_{2}\left(\tau_{*}, 0, \xi\right)$}

The following is the well-known criterion for the isomonodromic property.

Lемма 5.1. Suppose that the second-order ordinary differential equation

$$
\frac{\partial^{2}}{\partial z^{2}} f(z, \xi)+p(z, \xi) \frac{\partial}{\partial z} f(z, \xi)+q(z, \xi) f(z, \xi)=0
$$

is of Fuchsian type on $\mathbf{P}_{1}$ with the parameter $\xi$. The monodromy group for this equation is independent of the parameter $\xi$ if and only if there exist $a(z, \xi)$ and $b(z, \xi)$, which are rational in $z$, such that

$$
\frac{\partial}{\partial \xi} f(z, \xi)=a(z, \xi) \frac{\partial}{\partial z} f(z, \xi)+b(z, \xi) f(z, \xi) .
$$

By the above general criterion, to show that the monodromy matrix associated with the fundamental system $\hat{f}_{1}(z, \xi), \hat{f}_{2}(z, \xi)$ is independent of the parameter $\xi$, it suffices to show that $a(z, \xi)$ and $b(z, \xi)$, defined by

$$
a(z, \xi)=\frac{\left|\begin{array}{ll}
\hat{f}_{1 \xi}(z, \xi) & \hat{f}_{1}(z, \xi) \\
\hat{f}_{2 \xi}(z, \xi) & \hat{f}_{2}(z, \xi)
\end{array}\right|}{\left|\begin{array}{ll}
\hat{f}_{1 z}(z, \xi) & \hat{f}_{1}(z, \xi) \\
\hat{f}_{2 z}(z, \xi) & \hat{f}_{2}(z, \xi)
\end{array}\right|}, \quad b(z, \xi)=\frac{\left|\begin{array}{ll}
\hat{f}_{1 z}(z, \xi) & \hat{f}_{1 \xi}(z, \xi) \\
\hat{f}_{2 z}(z, \xi) & \hat{f}_{2 \xi}(z, \xi)
\end{array}\right|}{\left|\begin{array}{ll}
\hat{f}_{1 z}(z, \xi) & \hat{f}_{1}(z, \xi) \\
\hat{f}_{2 z}(z, \xi) & \hat{f}_{2}(z, \xi)
\end{array}\right|}
$$

are rational functions of $z$. Let

$$
g(z, \xi)=\frac{\left(2 \phi_{0}(\xi)+3 i \gamma \xi(2 z-1)\right)^{2}}{z^{4 / 3}(1-z)^{4 / 3}}
$$


then, by (4.9) and (4.10), the expression

$$
\widehat{f}_{2}(z, \xi)=\hat{f}_{1}(z, \xi) \int g(z, \xi) d z
$$

follows. Hence, we have

$$
\begin{aligned}
& \left|\begin{array}{ll}
\hat{f}_{1 z}(z, \xi) & \hat{f}_{1}(z, \xi) \\
\hat{f}_{2 z}(z, \xi) & \hat{f}_{2}(z, \xi)
\end{array}\right|=-\widehat{f}_{1}(z, \xi)^{2} g(z, \xi), \\
& \left|\begin{array}{ll}
\hat{f}_{1 \xi}(z, \xi) & \hat{f}_{1}(z, \xi) \\
\hat{f}_{2 \xi}(z, \xi) & \hat{f}_{2}(z, \xi)
\end{array}\right|=-\widehat{f}_{1}(z, \xi)^{2} \int g_{\xi}(z, \xi) d z .
\end{aligned}
$$

Thus

$$
a(z, \xi)=\frac{\int g_{\xi}(z, \xi) d z}{g(z, \xi)}
$$

follows. On the other hand, we immediately have

$$
b(z, \xi)=-\frac{\hat{f}_{1 z}(z, \xi)}{\hat{f}_{1}(z, \xi)} a(z, \xi)+\frac{\widehat{f}_{1 \xi}(z, \xi)}{\hat{f}_{1}(z, \xi)} .
$$

We have

$$
\begin{aligned}
& \frac{\hat{f}_{1 z}(z, \xi)}{\hat{f}_{1}(z, \xi)}=\frac{\partial}{\partial z} \log \hat{f}_{1}(z, \xi)=\frac{1-2 z}{3 z(1-z)}-\frac{6 i \gamma \xi}{2 \phi_{0}(\xi)+3 i \gamma \xi(2 z-1)} \\
& \frac{\hat{f}_{1 \xi}(z, \xi)}{\hat{f}_{1}(z, \xi)}=\frac{\partial}{\partial \xi} \log \hat{f}_{1}(z, \xi)=-\frac{2 \phi_{0}^{\prime}(\xi)+6 i \gamma z}{2 \phi_{0}(\xi)+3 i \gamma \xi(2 z-1)}
\end{aligned}
$$

These are both rational functions of $z$. Hence, if $a(z, \xi)$ is a rational function of $z$, then $b(z, \xi)$ is also a rational function of $z$. Therefore, we have the following lemma.

LEMma 5.2. The family $\widehat{\mathrm{DL}}_{2}\left(\tau_{*}, 0, \xi\right)$ is isomonodromic if and only if the integral constant of the indefinite integral

$$
G(z, \xi)=\left(z-z^{2}\right)^{1 / 3} \int \frac{4 \phi_{0}(\xi) \phi_{0}^{\prime}(\xi)-9 \xi g_{3}\left(\tau_{*}\right)(1-2 z)^{2}}{\left(z-z^{2}\right)^{4 / 3}} d z
$$

is determined so that $G(z, \xi)$ is the rational function of $z$ for all $\xi$.

Proof. By direct calculation, we have

$$
\begin{aligned}
a(z, \xi)=\frac{z-z^{2}}{\left(2 \phi_{0}-3 i \gamma \xi(1-2 z)\right)^{2}}\{ & 2 G(z, \xi)-12 i \gamma\left(\phi_{0}(\xi)\right. \\
& \left.\left.+\xi \phi_{0}^{\prime}(\xi)\right)\left(z-z^{2}\right)^{1 / 3} \int \frac{1-2 z}{\left(z-z^{2}\right)^{4 / 3}} d z\right\} .
\end{aligned}
$$


On the other hand, we have

$$
\int \frac{1-2 z}{\left(z-z^{2}\right)^{4 / 3}} d z=\left(\frac{3}{z-1}-\frac{3}{z}\right)\left(z-z^{2}\right)^{2 / 3}+\text { const. }
$$

This completes the proof.

Next we have the following lemma.

Lemma 5.3. The integral constant of the indefinite integral

$$
\left(z-z^{2}\right)^{1 / 3} \int \frac{z^{2}+c}{\left(z-z^{2}\right)^{4 / 3}} d z
$$

can be determined so that it is the rational function of $z$ if and only if $c=-1$.

Proof. Firstly, suppose $c=-1$, then we immediately have

$$
\int \frac{z^{2}-1}{\left(z-z^{2}\right)^{4 / 3}} d z=\frac{3\left(z-z^{2}\right)^{2 / 3}}{z}+\alpha
$$

Hence, putting $\alpha=0$, we have

$$
\left(z-z^{2}\right)^{1 / 3} \int \frac{z^{2}-1}{\left(z-z^{2}\right)^{4 / 3}} d z=3(1-z)
$$

Secondly, by the above, we have

$$
\left(z-z^{2}\right)^{1 / 3} \int \frac{z^{2}+c}{\left(z-z^{2}\right)^{4 / 3}} d z=(c+1)\left(z-z^{2}\right)^{1 / 3} \int \frac{1}{\left(z-z^{2}\right)^{4 / 3}} d z+3(1-z) .
$$

Let

$$
p(z)=\left(z-z^{2}\right)^{1 / 3} \int \frac{1}{\left(z-z^{2}\right)^{4 / 3}} d z
$$

then we have

$$
\frac{\partial}{\partial z} \log p(z)=\frac{1-2 z}{3\left(z-z^{2}\right)}+\frac{1}{\left(z-z^{2}\right) p(z)} .
$$

This implies that

$$
z(z-1) \frac{\partial}{\partial z} p(z)=\frac{1}{3}(2 z-1) p(z)-1
$$

Assume that one can choose the integration constant so that $p(z)$ is the rational function. Then there exist the polynomials $p_{1}(z), p_{2}(z)$, and $p_{3}(z)$ such that $p_{1}(0)=p_{2}(0)=0$ and

$$
p(z)=p_{1}\left(\frac{1}{z}\right)+p_{2}\left(\frac{1}{z-1}\right)+p_{3}(z) .
$$


Let

$$
p_{1}\left(\frac{1}{z}\right)=\sum_{j=1}^{l} \frac{\alpha_{j}}{z^{j}}, \quad p_{2}\left(\frac{1}{z-1}\right)=\sum_{j=1}^{m} \frac{\beta_{j}}{(z-1)^{j}}, \quad p_{3}(z)=\sum_{j=0}^{n} \gamma_{j} z^{j},
$$

then we have

$$
\begin{aligned}
& z(z-1) \frac{\partial}{\partial z} p_{1}\left(\frac{1}{z}\right)-\frac{1}{3}(2 z-1) p_{1}\left(\frac{1}{z}\right) \\
& =-\sum_{j=1}^{l} \frac{j \alpha_{j}}{z^{j-1}}+\sum_{j=1}^{l} \frac{j \alpha_{j}}{z^{j}}-\sum_{j=1}^{l} \frac{2}{3} \frac{\alpha_{j}}{z^{j-1}}+\sum_{j=1}^{l} \frac{1}{3} \frac{\alpha_{j}}{z^{j}}=c_{1}, \\
& z(z-1) \frac{\partial}{\partial z} p_{2}\left(\frac{1}{z-1}\right)-\frac{1}{3}(2 z-1) p_{2}\left(\frac{1}{z-1}\right) \\
& =\left((z-1)^{2}+(z-1)\right) \frac{\partial}{\partial z} p_{2}\left(\frac{1}{z-1}\right)-\frac{1}{3}(2(z-1)+1) p_{2}\left(\frac{1}{z-1}\right) \\
& =-\sum_{j=1}^{m} \frac{j \beta_{j}}{(z-1)^{j-1}}-\sum_{j=1}^{m} \frac{j \beta_{j}}{(z-1)^{j}}-\sum_{j=1}^{l} \frac{2}{3} \frac{\beta_{j}}{(z-1)^{j-1}}-\sum_{j=1}^{l} \frac{1}{3} \frac{\beta_{j}}{(z-1)^{j}}=c_{2}, \\
& z(z-1) \frac{\partial}{\partial z} p_{3}(z)-\frac{1}{3}(2 z-1) p_{3}(z) \\
& =\sum_{j=1}^{n} j \gamma_{j} z^{j+1}-\sum_{j=1}^{m} j \gamma_{j} z^{j}-\sum_{j=0}^{m} \frac{2}{3} \gamma_{j} z^{j+1}+\sum_{j=0}^{m} \frac{1}{3} \gamma_{j} z^{j}=c_{3},
\end{aligned}
$$

where $c_{1}+c_{2}+c_{3}=-1$. By these relations, we have

$$
l \alpha_{l}-\frac{1}{3} \alpha_{l}=0
$$

Hence $\alpha_{l}=0$ follows. Moreover, one verifies that

$$
\begin{gathered}
-j \alpha_{j}+(j-1) \alpha_{j-1}-\frac{2}{3} \alpha_{j}+\frac{1}{3} \alpha_{j-1}=0, \quad j=2,3, \ldots, l, \\
-\alpha_{1}-\frac{2}{3} \alpha_{1}=c_{1} .
\end{gathered}
$$

These imply that $\alpha_{l}=\alpha_{l-1}=\cdots=\alpha_{1}=0$, that is, $p_{1}(z)=0$ and $c_{1}=0$. Similarly, one can show that $p_{2}(z)=0$ and $c_{2}=0$. On the other hand, we have

$$
n \gamma_{n}-\frac{2}{3} \gamma_{n}=0
$$

Hence $\gamma_{n}=0$ follows. Moreover, we have

$$
\begin{aligned}
j \gamma_{j}-(j+1) \gamma_{j+1} & -\frac{2}{3} \gamma_{j}+\frac{1}{3} \gamma_{j+1}=0, \quad j=1,2, \ldots, n-1, \\
& -\gamma_{1}-\frac{2}{3} \gamma_{0}+\frac{1}{3} \gamma_{1}=0 .
\end{aligned}
$$


These imply that $\gamma_{n}=\gamma_{n-1}=\cdots=\gamma_{0}=0$. On the other hand, we have

$$
-\frac{1}{3} \gamma_{0}=c_{3}
$$

Hence $c_{3}=0$ follows. This is contradiction. This completes the proof.

Let

$$
K=-\frac{4 \phi_{0}(\xi) \phi_{0}^{\prime}(\xi)}{9 \xi g_{3}\left(\tau_{*}\right)}
$$

then we have

$$
\begin{aligned}
G(z, \xi) & =-9 \xi g_{3}\left(\tau_{*}\right)\left(z-z^{2}\right)^{1 / 3} \int \frac{(1-2 z)^{2}-K}{\left(z-z^{2}\right)^{4 / 3}} d z \\
& =-9 \xi g_{3}\left(\tau_{*}\right)\left(z-z^{2}\right)^{1 / 3}\left(2 \int \frac{1-2 z}{\left(z-z^{2}\right)^{4 / 3}} d z+4 \int \frac{z^{2}-(K+1) / 4}{\left(z-z^{2}\right)^{4 / 3}} d z\right) .
\end{aligned}
$$

Hence, we can determine the integral constant so that $G(z, \xi)$ is the rational function of $z$ if and only if $K=3$. Since we assumed that $\phi_{0}(0) \neq 0$, we have

$$
\phi_{0}(\xi)=\left(-\frac{27}{4} g_{3}\left(\tau_{*}\right) \xi^{2}+c\right)^{1 / 2}, \quad c \neq 0 .
$$

Thus, we proved the following theorem.

Theorem 5.4. Suppose $\tau_{*} \in \Theta_{2}$. Let $\phi_{0}(\xi)$ be defined as in (5.30). Then, the monodromy group for $\widehat{\mathrm{DL}}_{2}\left(\tau_{*}, 0, \xi\right)$ is isomorphic to that for Gauss' hypergeometric differential equation (4.5) for every $\xi \in \mathbf{P}_{1}$.

\section{Monodromy group of $\widehat{\mathrm{DL}}_{2}\left(\tau_{*}, 0,0\right)$}

By Theorem 5.4, to carry out the calculation of the monodromy group of $\widehat{\mathrm{DL}}_{2}\left(\tau_{*}, 0, \xi\right)$, it suffices to do it for $\widehat{\mathrm{DL}}_{2}\left(\tau_{*}, 0,0\right)$.

We denote $D=\mathbf{P}_{1} \backslash\{0,1, \infty\}$ and let $\pi_{1}\left(D, z_{0}\right)$ be the fundamental group of $D$ with the base point $z_{0} \in D$. Let

$$
y_{1}(z)=\widehat{f}(z), \quad y_{2}(z)=z \hat{f}^{\prime}(z)-\frac{1}{3} \hat{f}(z),
$$

and $X(z)={ }^{t}\left(y_{1}(z), y_{2}(z)\right)$. Then the Okubo form [4, page 177] of the Gauss' hypergeometric differential equation (4.5) is expressed as

$$
(z-B) \frac{\partial}{\partial z} X=A X
$$

where

$$
B=\left(\begin{array}{ll}
0 & 0 \\
0 & 1
\end{array}\right), \quad A=\left(\begin{array}{cc}
1 / 3 & 1 \\
4 / 9 & -2 / 3
\end{array}\right) .
$$


Let $\chi\left(z, z_{0}\right)=\left(X_{1}(z), X_{2}(z)\right)$ be the fundamental system of solutions of the Okubo form (6.2) near $z_{0}$. On the other hand, let $\gamma \in \pi_{1}\left(D, z_{0}\right)$ and let $\chi^{\gamma}\left(z, z_{0}\right)$ be the analytic continuation along the closed path $\gamma$. Then there exists $M_{\gamma} \in \mathrm{GL}(2, \mathbb{C})$ such that $\chi^{\gamma}\left(z, z_{0}\right)=$ $\chi\left(z, z_{0}\right) M_{\gamma}$. The map $\mu_{\chi}: \pi_{1}\left(D, z_{0}\right) \rightarrow \mathrm{GL}(2, \mathbb{C})$ defined by $\mu_{\chi}(\gamma)=M_{\gamma}$ is the linear representation of the fundamental group $\pi_{1}\left(D, z_{0}\right)$. The image $G=\mu_{\chi}\left(\pi_{1}\left(D, z_{0}\right)\right)$ is called the monodromy group associated with the fundamental system $\chi\left(z, z_{0}\right)$.

Let $F(\alpha, \beta, \gamma ; z)$ be the hypergeometric function. According to [4, pages 178-179], define the holomorphic solutions $Y(z, a)$ and the nonholomorphic solutions $X(z, a)$, for $a=0,1$, as follows:

$$
\begin{gathered}
Y(z, 0)={ }^{t}\left(-\frac{1}{3} F\left(1,-\frac{2}{3}, \frac{2}{3} ; z\right), F\left(1,-\frac{2}{3},-\frac{1}{3} ; z\right)\right), \\
X(z, 0)={ }^{t}\left(z^{1 / 3} F\left(\frac{4}{3},-\frac{1}{3}, \frac{4}{3} ; z\right),-\frac{1}{3} z^{1 / 3} F\left(\frac{7}{3}, \frac{2}{3}, \frac{7}{3} ; z\right)\right), \\
Y(z, 1)={ }^{t}\left(F\left(1,-\frac{1}{3}, \frac{2}{3} ; 1-z\right), \frac{9}{4} F\left(1,-\frac{1}{3},-\frac{1}{3} ; 1-z\right)\right), \\
X(z, 1)={ }^{t}\left(3(z-1)^{1 / 3} F\left(-\frac{1}{3}, \frac{4}{3}, \frac{1}{3} ; 1-z\right),(z-1)^{-2 / 3} F\left(-\frac{4}{3}, \frac{1}{3},-\frac{2}{3} ; 1-z\right)\right) .
\end{gathered}
$$

The matrix functions $(Y(z, 0), X(z, 0))$ and $(Y(z, 1), X(z, 1))$ are both the fundamental systems of solutions of the Okubo form (6.2) defined near $z=0$ and $z=1$, respectively. By the method explained precisely in [4, pages 193-199], using these fundamental systems, one can solve the connection problem and finally obtain the generators $M_{0}$ and $M_{1}$ of the monodromy group $G$ as follows:

$$
M_{0}=\left(\begin{array}{cc}
\exp \left(\frac{2}{3} \pi i\right) & \exp \left(\frac{2}{3} \pi i\right)-1 \\
0 & 1
\end{array}\right), \quad M_{1}=\left(\begin{array}{cc}
1 & 0 \\
-\exp \left(-\frac{1}{3} \pi i\right)-1 & 1
\end{array}\right)
$$

It is easy to see that the monodromy group of the Okubo form (6.2) coincides with that of Gauss' hypergeometric equation (4.5).

\section{Acknowledgment}

This work was partially supported by Grant-in-Aid for Scientific Research (C) 13640195 from the Japanese Ministry of Education, Culture, Sports, Science and Technology.

\section{References}

[1] J. G. Darboux, Sur une proposition relative aux équations linéaires, C. R. Acad. Sci. Paris 94 (1882), 1456-1459 (French).

[2] F. Gesztesy and H. Holden, Darboux-type transformations and hyperelliptic curves, J. Reine Angew. Math. 527 (2000), 151-183.

[3] F. Gesztesy and R. Weikard, Spectral deformations and soliton equations, Differential Equations with Applications to Mathematical Physics (W. F. Ames, E. M. Harrell II, and J. V. Herod, eds.), Math. Sci. Engrg., vol. 192, Academic Press, Massachusetts, 1993, pp. 101-139.

[4] M. Kohno, Global Analysis in Linear Differential Equations, Mathematics and Its Applications, vol. 471, Kluwer Academic Publishers, Dordrecht, 1999. 
[5] M. Ohmiya, KdV polynomials and $\Lambda$-operator, Osaka J. Math. 32 (1995), no. 2, 409-430.

[6] _ Spectrum of Darboux transformation of differential operator, Osaka J. Math. 36 (1999), no. 4, 949-980.

[7] M. Ohmiya and M. Urakubo, Isospectral property of double Darboux transformation, in preparation.

Mayumi Ohmiya: School of Engineering, Doshisha University, Kyotanabe 610-0394, Japan

E-mail address: momiya@mail.doshisha.ac.jp 


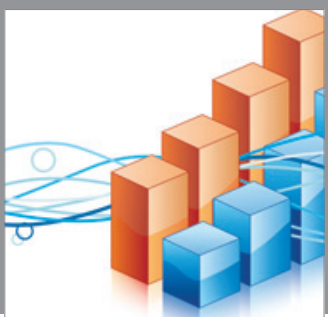

Advances in

Operations Research

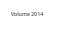

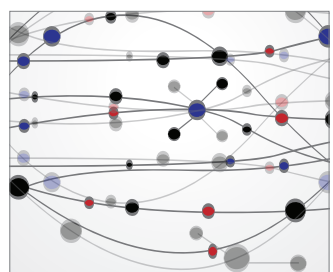

\section{The Scientific} World Journal
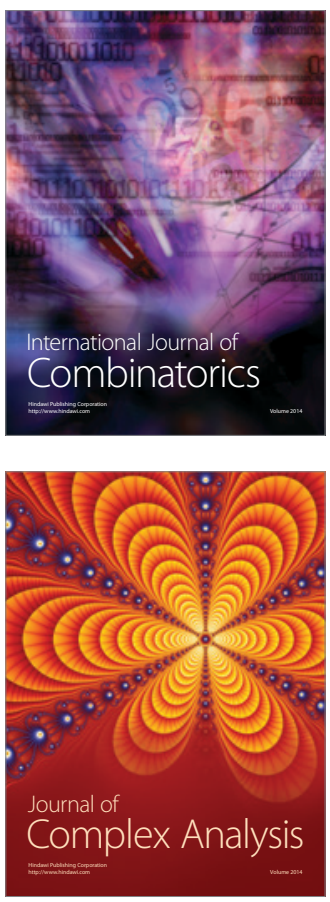

International Journal of

Mathematics and

Mathematical

Sciences
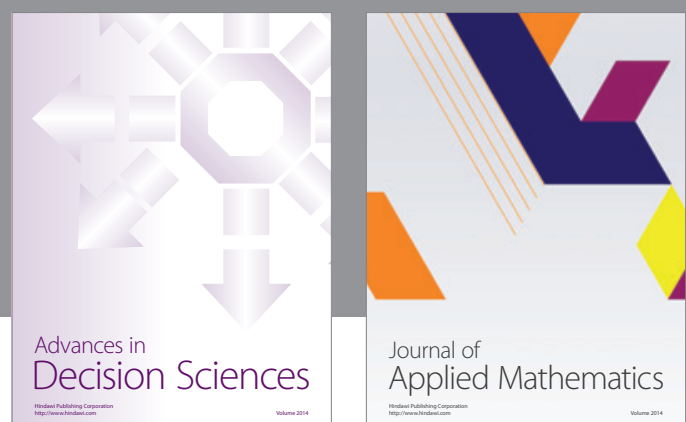

Journal of

Applied Mathematics
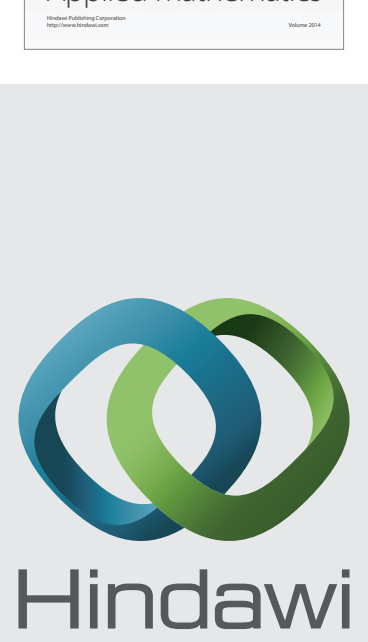

Submit your manuscripts at http://www.hindawi.com
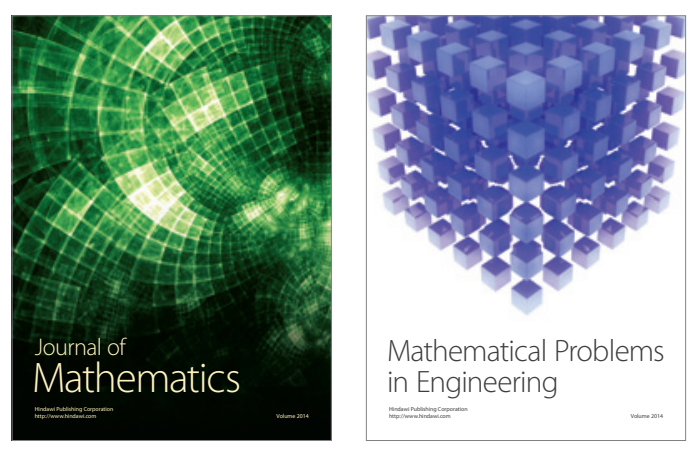

Mathematical Problems in Engineering
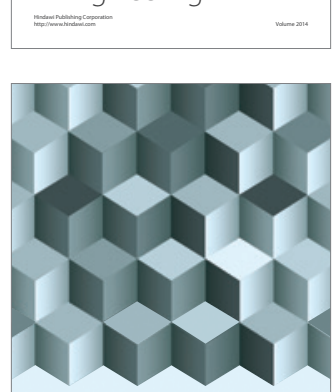

Journal of

Function Spaces
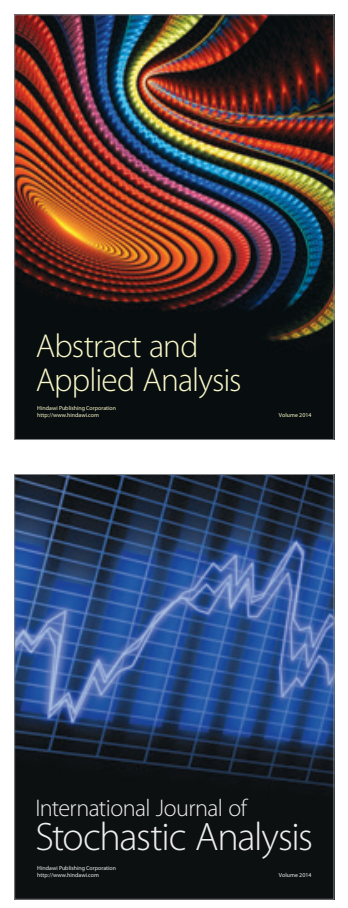

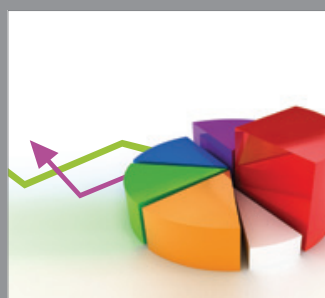

ournal of

Probability and Statistics

Promensencen
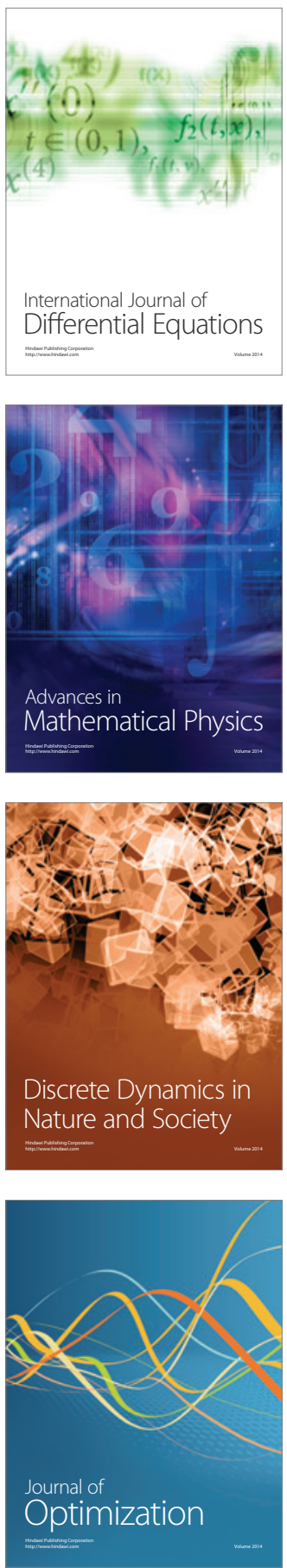